\title{
Dual Port Cognitive Radio Antenna Using Tunable Band Pass Filter
}

\author{
Nishant Kumar \\ Assistant professor, Dept. of EXTC, Sardar Patel Institute of Technology, Mumbai, India
}

\begin{abstract}
:
In this paper a dual port microstrip antenna with tunable band pass filter is proposed for cognitive radio applications. In single port reconfigurable antennas for cognitive radio, sensing and communication is done simultaneously. This can lead to failure of real time communication, also it may induce interference to primary user, dual antenna system solves this problem. The proposed antenna consist of one UWB microstrip antenna for sensing the holes in spectrum and other is communication antenna. Communication antenna is made tunable by using varacter diode in ' $G$ ' shaped DMS(defected microstrip structure) filter integrated in feedline.The sensing antenna is having UWB bandwidth from $3.4 \mathrm{GHz}$ to $13.2 \mathrm{GHz}$ and efficiency of more than $80 \%$. The narrowband antenna has dual and triple operating frequencies which is tunable in the range of 4-5 GHz, 6-10 GHz and 10-11 GHz according to the biasing of varacterdiode. This antenna as efficiency more than $70 \%$.
\end{abstract}

\section{KEYWORDS:}

ultra wideband (UWB) antenna, Defected microstripstructure (DMS), Tunable filter,varacter diode

\section{INTRODUCTION}

As the communication industry is growing the users and the need for higher data rate is also increasing. The limited spectrum band and current band allocation techniques are not able to fulfil this need. The current band sharing scheme assigns particular band to a communication service which are not dynamic and a licensed user can only use the band allocated to them. Software defined radio is future technology which will solve this problem by dynamic sharing of spectrum, as the unlicensed users(secondary users) can access the licensed band of primary user in opportunistic way without having any interference with them. Spectrum underlay and spectrum overlay are the two techniques that can be used for dynamic sharing of spectrum. In underlay cognitive radio (CR) system the secondary user uses spectrum of primary user under the noise floor, so the transmission power used by secondary user is restricted(less than $-42 \mathrm{dBm} / \mathrm{MHz}$ in the 3.1-10.6 GHz band).[3] In overlay cognitive system the secondary user searches unused band of primary user for communication without interference. The users in CR system must follow some requirements in order to communicate without interference and have good quality of services.

- Spectrum sensing: - determine the part of spectrum of licensed user which is not been used and is available.

- Spectrum decision: - select best vacant channel.

- Spectrum sharing: - Manage access to this channel with other users.

- Spectrum mobility: - when licensed user need that channel then vacate it. 
To fulfil the above requirements cognitive radio has to be reconfigurable, self-organised, software defined and cognitive. Cognitive capability means the system has to sense the entire channel and find out the best available channel which is not being used by the primary user. Reconfigurable capability enables the system to dynamically change its operating frequency and adapt its modulation process and transmit power. Self-organised means the system has the ability of good connection management and mobility, it also ensures security to the spectrum sharing. In overlay cognitive radio system secondary user has to use a UWB antenna to sense the entire spectrum and choose the unused band where it can transmit or receive communication signals. In this process the unlicensed user has to manage its band requirements and see when and where to transmit its communication signal rather than restrict power of transmission. Most important thing is this is to use better filter techniques so that the signals used should not interfere with licensed user, and also the dynamic management system should sense the channel every defined time to see if that spectrum is being demanded by licensed user. If that spectrum is demanded by licensed user than the secondary user has to sense again the channel and change its operating frequency to new white space (hole) and this process continuous.[10]

The approach in this paper is to make continuous sensing of the channel and parallel communication process. This approach needs two antenna one is UWB antenna with omnidirectional pattern for sensing and other is reconfigurable narrowband by which communication can take place. As shown in figure 1.

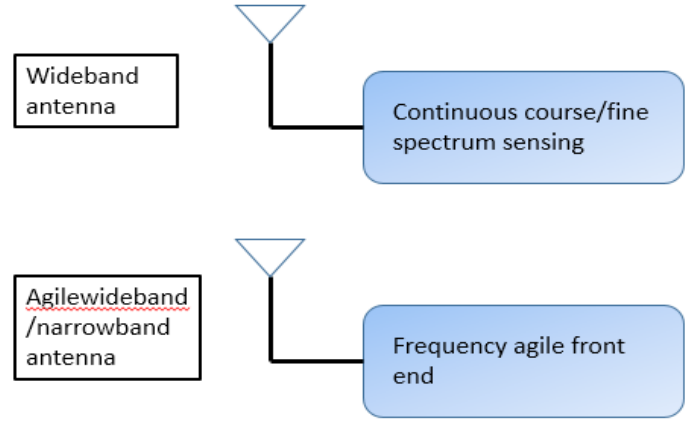

Figure 1: parallel sensing and communication process in CR system [1]

The recent developments in cognitive radio system has encouraged new challenges in reconfigurable antenna design which can dynamically change the operating frequency keeping radiation pattern and gain to be same.Many designs have been proposed in literature which make use of Active switches(like PIN diodes,RFMEMS, Varactor diodes etc),Optical switches, mechanical switches.[1-2][6-9] In this paper tunability is achieved by using varactor diode. [4]

Varactor diodes are also known as varicaps and its small junction capacitance varies by applied DC bias voltage as given in equation 1.it has continuous tuning ability and has wide applications in RF and microwave applications. The varactor diode is placed across ' $G$ ' slot in feedline of narrowband antenna.

$$
C_{j}(V)=\frac{C_{0}}{\left(1-\frac{V}{V_{0}}\right)^{\gamma}}
$$




\section{ANTENNA GEOMETRY}

Figure 2 (front view) and figure 3(rear view) shows the schematic of the proposed antenna. It consist of two square patches with microstrip feedline. The substrate used in this design is FR4epoxy with dielectric constant 4.3 , thickness is $1.6 \mathrm{~mm}$ and loss tangent of 0.0018 . The optimized parameters of the proposed design after manual optimization using initial formulas and CST design studio are as follows: for UWB antenna $\mathrm{R}=10.5, \mathrm{~W}=55, \mathrm{~L}=34, \mathrm{wp} 1=11.8, \mathrm{lp} 1=10.5$, $\mathrm{lg}=17.6, \mathrm{wf}=3.2$; for reconfigurable antenna $\mathrm{lp} 2=9.42, \mathrm{wp} 2=13.14, \mathrm{~g}=0.6$; parameters for ground plane are $\mathrm{Wg} 1=12, \mathrm{Wg} 2=22, \mathrm{Wg} 3=12$; and the filter design parameters are $\mathrm{la}=3.14, \mathrm{lb}=4.6, \mathrm{lc}=6.95, \mathrm{ld}=2.925, \mathrm{wa}=0.8, \mathrm{wb}=0.4, \mathrm{wc}=0.8$.

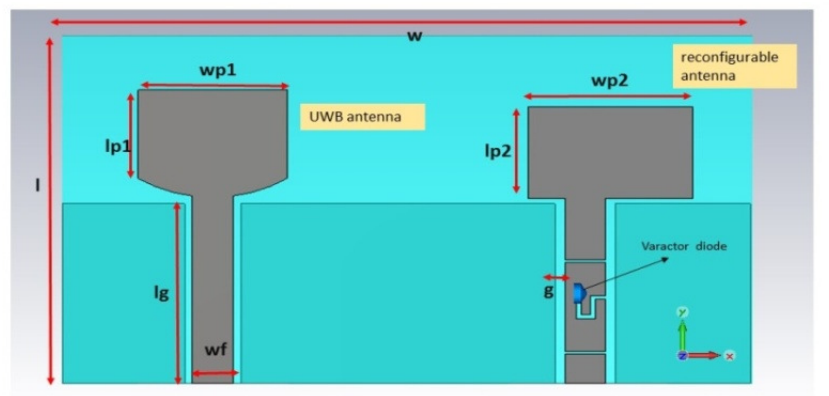

Figure 2. Front view of dual port antenna

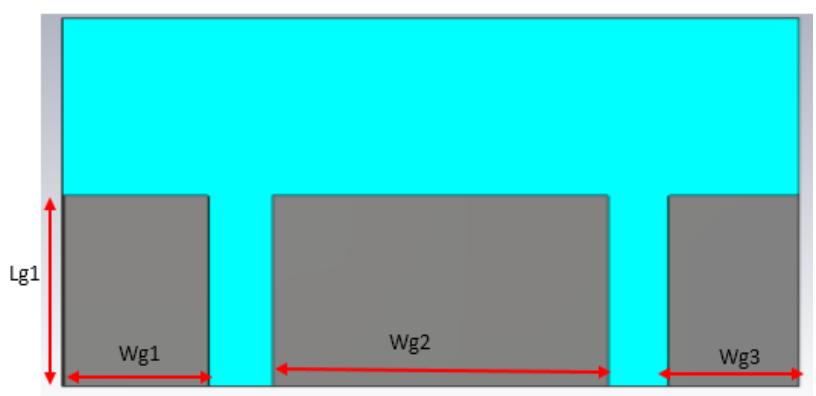

Figure 3: Rear view of the antenna

The reconfiguration in the narrowband antenna is created by embedding $\mathrm{G}$ shaped filter with two parallel slots and a varactor for tuning in feedline. . The bandpass defected microstrip structure (DMS) filter is shown in figure 4 and its equivalent circuit in figure 5.[8]

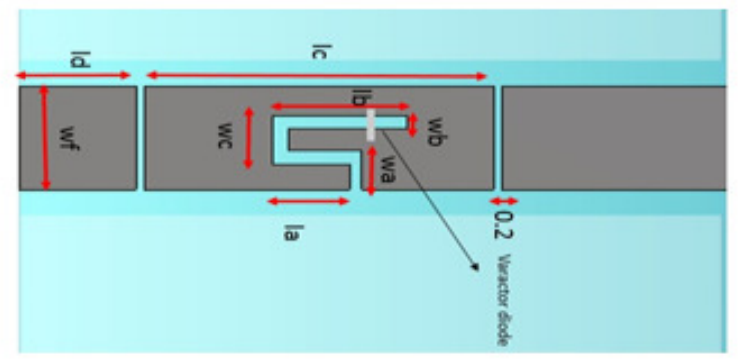

Figure 4:Design of proposed DMS filter in the feedline 
International Journal Of Microwave Engineering (JMICRO) Vol.1, No.2, April 2016

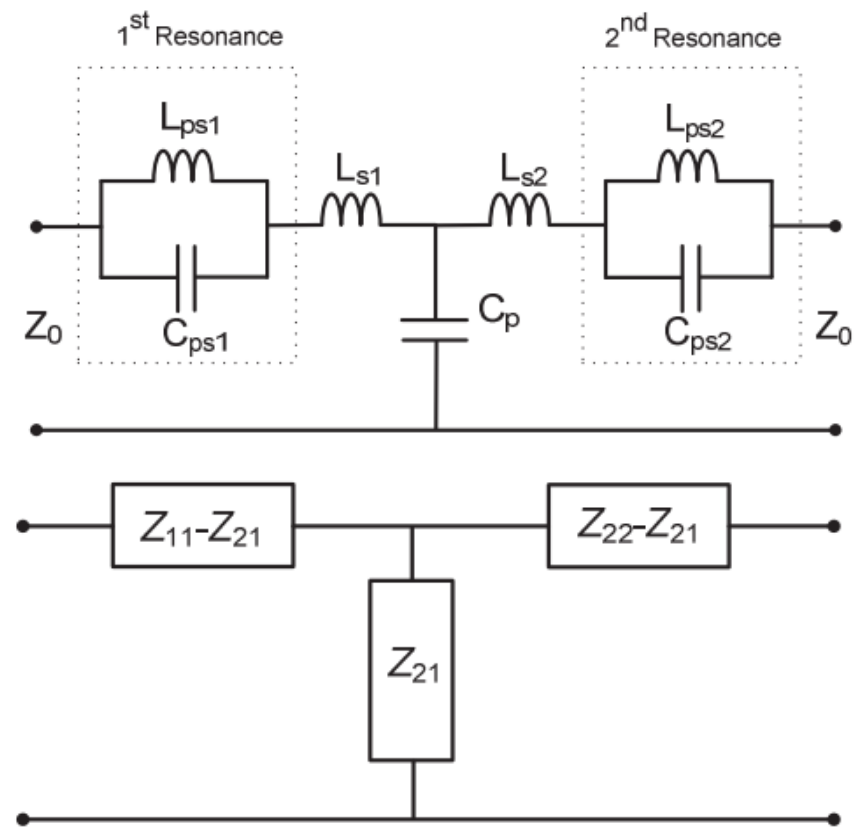

Figure 5: equivalent circuit diagram of $\mathrm{G}$ shaped filter

G shaped band stop filter is modelled by two LC resonator as shown in figure 5. The values of LC resonator can be extracted by EM simulation, the equation for Lpsi and Cpsi is given below, these values are derived from transmission parameters S21 of a port. [5],[6]

$$
\begin{gathered}
C_{p s i}=\frac{1}{4 \pi Z_{0} \Delta_{3 d B_{-} f 0 i}} \text { for } i=1,2 \\
L_{p s i}=\frac{1}{\left(2 \pi f_{0 i}\right)^{2} C_{p s i}} \text { for } i=1,2
\end{gathered}
$$

Where,

$Z_{0}=$ characteristic impedance of the network port

$f_{0 i}=$ First and second resonant frequency.

$C_{p s i}=$ capacitance of the equivalent circuit

$L_{p s i}=$ inductance of the equivalent circuit

The remaining parameters are calculated by matching $\mathrm{Z}$ network

$$
\begin{aligned}
C_{p} & =-\frac{1}{2 \pi f_{T} X_{21}} \\
L_{s i} & =-\frac{X_{i i}-X_{21}}{2 \pi f_{T}}+\frac{L_{p s i}}{\left(\frac{f_{T}}{f_{0 i}}\right)^{2}-1} \text { for } \mathrm{i}=1,2
\end{aligned}
$$




\section{Simulation results and discussions}

The proposed antenna is dual port, one antenna is giving UWB bandwidth from $3.4 \mathrm{GHz}$ to 13.2 $\mathrm{GHz}$.the reconfigurable antenna is giving dual band or triple band with different resonating frequencies according to the values of capacitance of varactor diode. Figure 6 shows the UWB antenna reflection coefficient curve and isolation coefficient curve, i.e. is below $-15 \mathrm{~dB}$, that means if port 1 is given $1 \mathrm{~W}$ power than port 2 will receive below $0.03 \mathrm{~W}$ power, thus a good isolation is between them and they will not interfere each other. Figure 6 shows return loss curve of reconfigurable antenna with dual and triple resonating frequency, one is around $4 \mathrm{GHz}$ to 5 $\mathrm{GHz}$, second is from $6 \mathrm{GHz}$ to $10 \mathrm{GHz}$, and third is from $10 \mathrm{GHz}$ to $11 \mathrm{GHz}$.

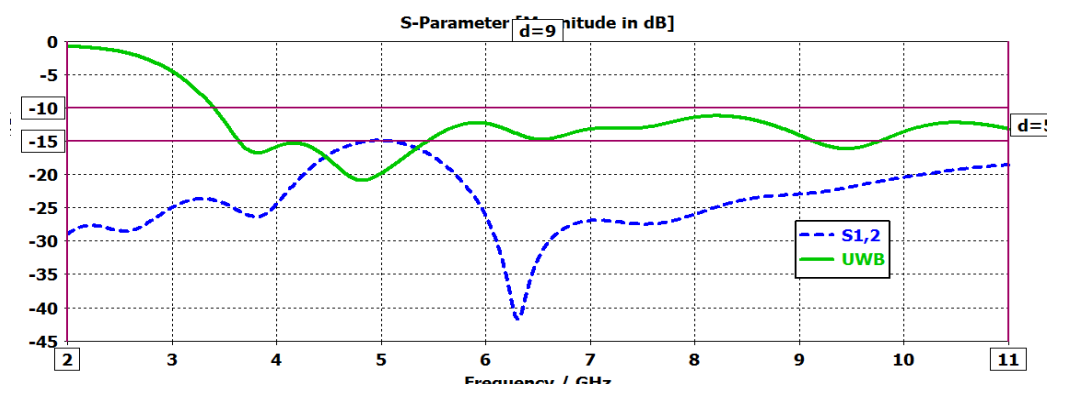

Figure 6: reflection coefficient and isolation coefficient curve for UWB antenna

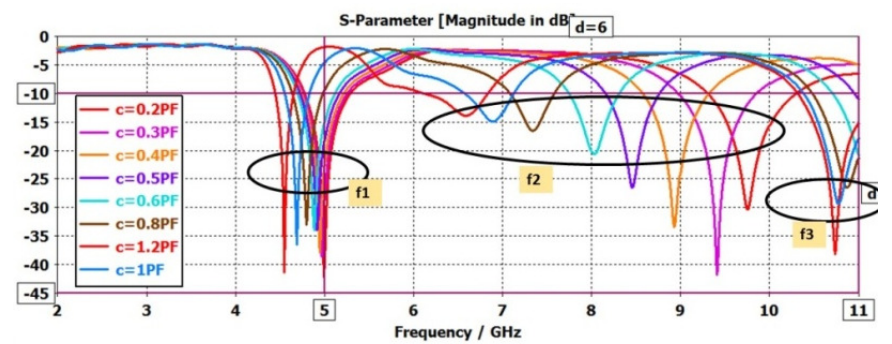

Figure 7: Reflection coefficient curve for reconfigurable antenna

It can be observed from figure 7 that second resonance frequency is most varying with varying the biasing voltage of the varactor diode. This is plotted below showing, as the reverse biased voltage of the varactor increased and hence increasing the capacitance, the second operating frequency decreases.

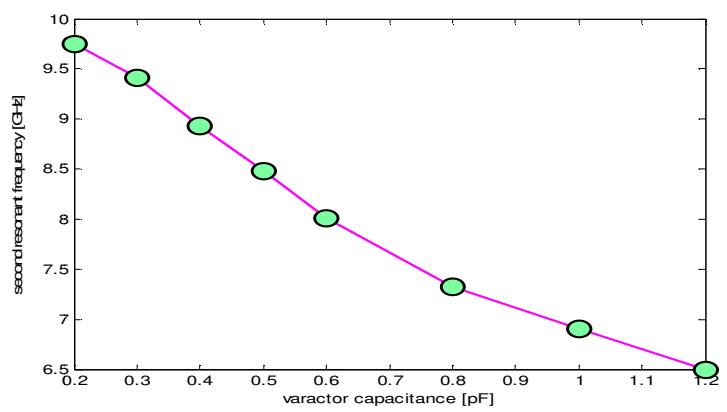

Figure 8: Variation of second resonating frequency Vs capacitance of varactordiode 
Table 1 given below shows the resonating frequency, gain and efficiency of UWB and reconfigurable antenna at different bias voltages to the varactor. It can be observed in case of UWB antenna good gain of more than $3 \mathrm{~dB}$ and efficiency more than $80 \%$ is achieved. In case of narrowband antenna gain of more than $2.7 \mathrm{~dB}$ and efficiency more than $70 \%$ is achieved. Figure 8 shows the variation of second resonance frequency with different capacitance values of varactor diodes.

Table 1: resonating frequency, gain and efficiency for different varactor capacitance

\begin{tabular}{|c|c|c|c|c|c|c|c|}
\hline \multirow[t]{3}{*}{ CASE } & \multirow{3}{*}{$\begin{array}{c}\text { Varactor } \\
\text { diode } \\
\text { capacitance } \\
\text { value (PF) }\end{array}$} & \multicolumn{2}{|c|}{ Resonating frequency } & \multicolumn{4}{|c|}{ Gain (dB) and efficiency (\%) } \\
\hline & & \multirow[t]{2}{*}{$\mathrm{fr} 1(\mathrm{GHz})$} & \multirow[t]{2}{*}{$\mathrm{fr} 2(\mathrm{GHz})$} & \multicolumn{2}{|c|}{ UWB } & \multicolumn{2}{|c|}{ Reconfigurable(fr2) } \\
\hline & & & & Gain & Efficiency & Gain & efficiency \\
\hline 1 & 0.2 & 5 & 9.71 & 4.47 & 80 & 5.37 & 75 \\
\hline 2 & 0.3 & 5 & 9.41 & 4.1 & 81 & 4.63 & 77 \\
\hline 3 & 0.4 & 4.94 & 8.93 & 3.64 & 82 & 3.56 & 75 \\
\hline 4 & 0.5 & 4.90 & 8.48 & 3.2 & 83 & 2.7 & 76 \\
\hline 5 & 0.6 & 4.87 & 8.01 & 2.86 & 84 & 2.75 & 79 \\
\hline 6 & 0.8 & 4.8 & 7.32 & 3.553 & 85 & 3.6 & 79 \\
\hline 7 & 1 & 4.68 & 6.9 & 4.1 & 86 & 3.57 & 78 \\
\hline 8 & 1.2 & 4.5 & 6.5 & 4.27 & 87 & 3.6 & 76 \\
\hline
\end{tabular}

The radiation pattern of UWB and reconfigurable antenna for four frequencies(a) $9.75 \mathrm{GHz}$ (b) $8.93 \mathrm{GHz}$ (c) $8.01 \mathrm{GHz}$ (d) $6.5 \mathrm{GHz}$ is shown in figure 9 and 10 . It shows it is omnidirectional as needed for a sensing and communication antennas for cognitive radio systems. The reconfigurable filter is embedded in feedline which does not distort the radiation pattern and this is one of the major advantage of this design. Red part is $\mathrm{E}$ plane i.e. $\mathrm{YZ}$ plane and black is $\mathrm{H}$ plane i.e. XZ plane.

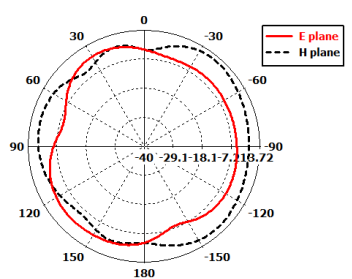

Theta / Degree vs. do

(a)

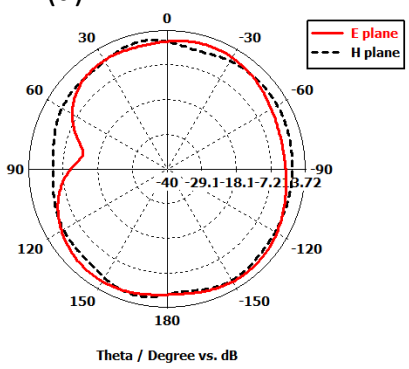

(c)

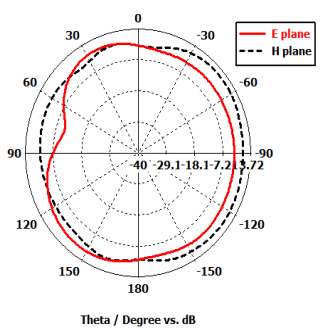

(b)

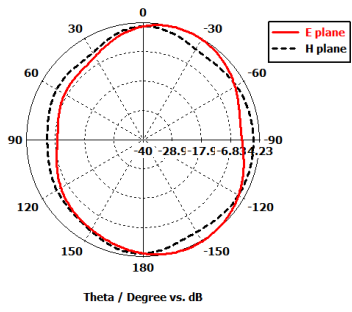

(d)

Figure 9: radiation pattern of UWB antenna at (a) $9.75 \mathrm{GHz}$ (b) $8.93 \mathrm{GHz}$ (c) $8.01 \mathrm{GHz}$

(d) $6.5 \mathrm{GHz}$ 


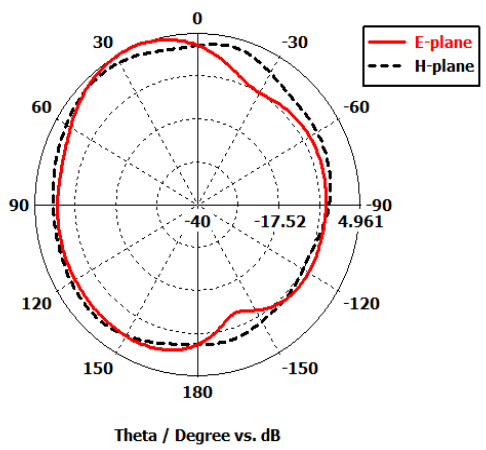

(a)

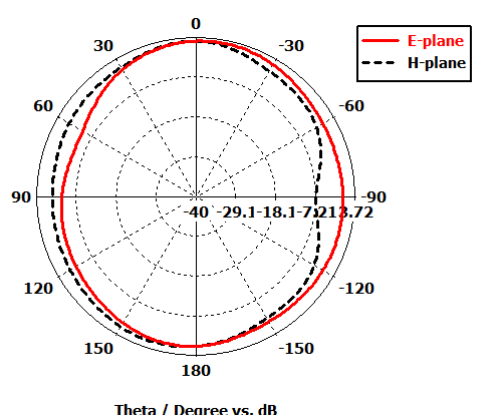

(c)

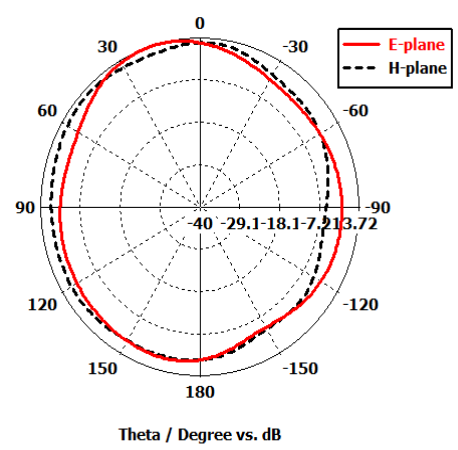

(b)

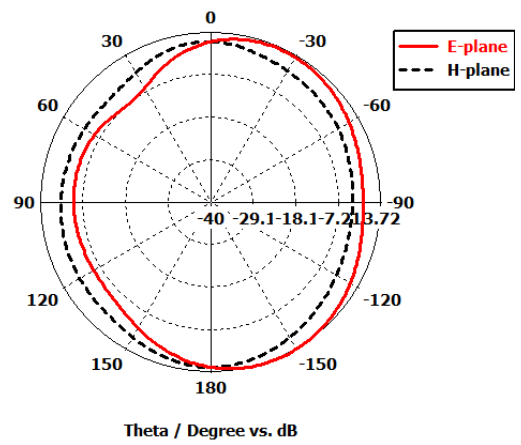

(d)

Figure 10: radiation pattern of reconfigurable antenna at (a) $9.75 \mathrm{GHz}$ (b) $8.93 \mathrm{GHz}$ (c) $8.01 \mathrm{GHz}$ (d) $6.5 \mathrm{GHz}$

\section{Conclusion}

A planar dual port antenna consisting of one UWB sensing antenna and other narrow-band tunable communication antenna for cognitive radio applications has been studied. The tunability is achieved by using varactor diode in the DMS ' $G$ ' shaped filter embedded in the feedline. The filter used here has many advantages over other structures like D.C bias circuit can be implemented easily and this does not disturb radiation pattern of the antenna. The UWB band for sensing holes are from $3.5 \mathrm{GHz}$ to $11 \mathrm{GHz}$ as given by $\mathrm{FCC}$ (federal communications commission ). The communication antenna has wide range of operating frequency from 4 to $11 \mathrm{GHz}$ depending on the varactor diode parameter. The radiation pattern is omni-directional as required and efficiency of both antennas are very good i.e. over 70\%.It is simulated in CST Microwave Studio software and validates the purpose of this antenna to be used in cognitive radio applications.

\section{REFERENCES}

[1] Yingsong Li; Wenxing Li; Mittra, R., "Integrated dual-purpose narrow/ultra-wide band antenna for cognitive radio applications," in Antennas and Propagation Society International Symposium (APSURSI), 2012 IEEE , vol., no., pp.1-2, 8-14 July 2012

[2] Ebrahimi, E.; Hall, P.S., "A dual port wide-narrowband antenna for cognitive radio," in Antennas and Propagation, 2009. EuCAP 2009. 3rd European Conference on , vol., no., pp.809-812, 23-27 March 2009

[3] Mohammed Al-Husseini , Karim Y. Kabalan, Ali El-Hajj and Christos G. Christodoulou, "Advancement in Microstrip Antennas with Recent Applications Chapter 14 "Edited by Ahmed Kishk, ISBN 978-953-51-1019-4, 394 pages, Publisher: Intec. 
[4] Brown, E.R., "RF-MEMS switches for reconfigurable integrated circuits," Microwave Theory and Techniques, IEEE Transactions on, vol.46, no.11, pp.1868,1880, Nov 1998.

[5] Kazerooni M, Cheldavi A, Kamarei M (2009),"A Novel Bandpass Defected Microstrip Structure (DMS) Filter for Planar Circuits." Proceedings of the 2009 Progress in Electromagnetics Research Symposium (PIERS 2009). Moscow, Russia. 1821 Aug 2009. pp.12141217.

[6] Ghanem, F.; Hall, P.S.; Kelly, J.R., "Two port frequency reconfigurable antenna for cognitive radios," Electronics Letters, vol.45, no.11, pp.534,536, May 212009

[7] Chaudhary, G.; Phirun Kim; YongchaeJeong; Jongsik Lim; Jaehoon Lee, "Analysis and circuit modeling method for defected microstrip structure in planar transmission lines," Microwave Conference Proceedings (APMC), 2011 Asia-Pacific , vol., no., pp.999,1002, 5-8 Dec. 2011

[8] Kumar, N.; Raju, P.A.; Behera, S.K., "Frequency reconfigurable microstrip antenna for cognitive radio applications," in Communications and Signal Processing (ICCSP), 2015 International Conference on , vol., no., pp.0370-0373, 2-4 April 2015

[9] Raju, P.A.; Kumar, N.; Behera, S.K., "Semi circular -semi hexagon microstrip antenna integrated with DRA for cognitive radio applications," in Communications and Signal Processing (ICCSP), 2015 International Conference on , vol., no., pp.0031-0034, 2-4 April 2015

[10] Hamid,F. Ghanem, F. J.Herraiz-Martinez, and D .Segovia Vargas, "Reconfigurable antenna challenges for future radio systems”,3rd Eur. Conf. Antennas Propa.,Berlin,Germany, 2009

[11] Tawk, Y.; Costantine, J.; Christodoulou, C.G., "Reconfigurable Filtennas and MIMO in Cognitive Radio Applications," in Antennas and Propagation, IEEE Transactions on , vol.62, no.3, pp.10741083, March 2014 